\title{
Acute and chronic paracetamol overdose in paediatric population: Protocol of a prospective study of cohort to evaluate clinic factor and biomarkers to predict development of hepatotoxicity.
}

\author{
Hoi Y. Tong ${ }^{1,5}$, Lucía Díaz García ${ }^{1}$, Santos García García², José Antonio Ruiz Domínguez², Julia Martín Sánchez², Pilar \\ Storch de Gracias ${ }^{3}$, Aris Rivas ${ }^{4}$, Mario Muñoz ${ }^{1}$, Rafael Hernández Zabala ${ }^{1}$, Irene García García ${ }^{1}$, Alberto M. Borobia \\ Pérez ${ }^{1,5}$, Antonio Carcas Sansuán ${ }^{1,5}$, José Carlos Martínez Ávila ${ }^{1}$, Elena Ramírez García ${ }^{1,5^{*}}$ \\ ${ }^{1}$ Department of Clinical Pharmacology, Hospital Universitario La Paz, IdiPAZ, School of Medicine, Universidad Autónoma de Madrid, Madrid, Spain. \\ 2Paediatric Emergency Department, Hospital Universitario La Paz, IdiPAZ, Madrid, Spain. \\ ${ }^{3}$ Paediatric Emergency Department, Hospital Universitario Niño Jesús, Madrid, Spain \\ ${ }^{4}$ Paediatric Emergency Department, Hospital Universitario Gregorio Marañón, Madrid, Spain \\ ${ }^{5} \mathrm{SCReN}$ (Spanish Clinical Research Network) \\ ${ }^{*}$ Corresponding author: \\ Elena Ramírez García Department of Clinical Pharmacology, Hospital Universitario La Paz, IdiPAZ, School of Medicine, Universidad Autónoma de \\ Madrid, Madrid, Spain E-mail: elena.ramirez@inv.uam.es
}

Background: In Spain, 30\% of cases of acute liver failure remaining undetermined. Paracetamol is the main drug causing acute liver failure in children of some countries like the United States, UK and other European countries. The key factors to assess in paracetamol toxicity, the ingested dose and the time from the poisoning, are difficult to assess in children where accidental acute poisoning or medication errors are not rare. Metabolomics technology may be able to identify specific biomarkers of toxicity and adverse events in early stages. The identification of paracetamol toxicity biomarkers could have important clinical implications for patients who can not apply the Rumack-Matthew nomogram and could be useful in the evaluation of children with acute liver failure of unknown etiology, and to predict liver damage before elevation of transaminases.

Methods and design: This is an observational prospective study of case control. The protocol was approved by the Clinical Research Ethics Committee of the La Paz University Hospital. It will recruit patients who will be attending in the emergency paediatric at La Paz University Hospital, at Niño Jesus University Hospital, and at Gregorio Marañón University hospital with suspected acute or chronic intake of paracetamol. Likewise, two cohorts of control will be recruited. It is expected to recruit at least 36 cases and 144 controls, matched for age and clinical characteristics. Peripheral blood, plasma, serum and urine samples will be collected, paracetamol serum concentrations, hepatic and renal function, coagulation and metabolomic analysis.

Discussion: It is important to determine the clinical factors and biomarkers that predict the development of hepatotoxicity in paediatric population following acute and chronic intake of paracetamol and develop a predictive model to assess the risk of hepatotoxicity in both type of intoxication by paracetamol suited to paediatric patients for use in clinical practice.

\section{Study registration:}

Protocol code: GEIPA-2012-01

AEMPS classification: EPA-OD

Study Reference Number of European Network of Centers for Pharmacoepidemiology and Pharmacovigilance: ENCePP: ENCePP/SDPP/3276

\footnotetext{
Keywords: Paracetamol, overdose, toxicity, hepatotoxicity, acute liver failure, paediatric population, biomarkers, pharmacokinetics, pharmacogenetics, metabolomics.

Received March 31, 2017 Accepted July 31, 2017 Published August 1, 2017.

Copyright: (c) 2017 Author. This is an open-access article distributed under the terms of the Creative Commons Attribution License, which permits unrestricted use, distribution, and reproduction in any medium, provided the original author and source are credited. Editor: Jesús Frías Iniesta

Cite as: Tong HY., Díaz García L., García García S., Ruiz Domínguez JA., Martín Sánchez J., Storch de Gracias P., Rivas A., Muñoz M., Hernández Zabala R., García García I., Borobia Pérez AM., Carcas Sansuán AJ., Martínez Ávila JC., Ramírez García E. Acute and chronic paracetamol overdose in paediatric population: Protocol of a prospective study of cohort to evaluate clinic factor and biomarkers to predict development of hepatotoxicity. IBJ Clin Pharmacol 2017 1(1):e0007.

Funding: The authors have no financial relationships relevant to this article to disclose.

Competing Interests: The authors declare no conflicts of interest.
} 


\section{BACKGROUND}

Paracetamol is the most widely drug used for the treatment of pain and fever, although it has a fairly acceptable safety profile when used at the recommended dose. In the overdose situation, it may cause hepatotoxicity and fulminant hepatic failure. It is the main drug causing acute liver failure in some countries such as the United States, United Kingdom and other European countries ${ }^{(1-3)}$. According to the report of the adverse event reporting system of FDA in the United States between 1998 and 2005, paracetamol ranked 5th among drugs suspected of causing any serious non-fatal and fatal adverse reactions ${ }^{(4)}$. Acute liver failure (ALF) is a rare case, which can compromise the patient's life or end up in transplantation in more than $45 \%$ of cases. It is reported that the cause of acute hepatic failure is not demonstrated among $40-50 \%$ of paediatric patients ${ }^{(5)}$. The key factors to assess in paracetamol toxicity, such as the ingested dose and the time from the poisoning, are difficult to assess in children where accidental acute poisoning or medication errors are not rare. Paracetamol represents an important cause that must be taken into account in the assessment of any child with acute liver failure. In a multicenter study that determined the causes of acute liver failure in children less than 17 years of age, it was obtained that $8.8 \%$ of the patients had a history of paracetamol intake ${ }^{(6)}$. It has been observed that most intoxications in young children are accidental by oral via. Intentional intoxications are more frequent among adolescents. In Spain, the exposure to a potentially toxic substance is a rare cause of consultation in the paediatric emergency, which occurs in less than $0.3 \%$ of all consultations, with drugs being the substances most commonly involved in different paediatric age groups ${ }^{(7)}$. In a retrospective study conducted by our group between 2005 and 2010, the incidence of paediatric paracetamol intoxication was 0.8 [Poisson-95\% confidence interval (CI): 0.03-3.69] per 10.000 inhabitants aged less than 18 years. The most common cause of poisoning was attempted suicide $(47.8 \%)$ in teenagers with a median of age of 15 years, followed by accidental poisoning $(42.2 \%)$ in babies with a median of age of 2.65 years ${ }^{(8)}$.

The public health impact of paracetamol overdose has been evaluated in several epidemiological studies. A multicenter prospective study of patients younger than 18 years of age conducted in the Unites States between 1999 and 2004 found that of a total of 348 patients with ALF criteria, $14 \%$ were due to acute paracetamol poisoning ${ }^{(5)}$. The results are in contrast to data from a multicenter study in Spain, in which paracetamol was considered a cause of acute liver failure in only $2.2 \%$ of 267 cases identified between 1992 and 2000 and the estimated incidence of acute liver failure during the study period was 1.4 cases per million of habitants per year, although the origin of $30 \%$ of cases remains undetermined ${ }^{(9)}$. However, it is striking that the oral solution formulations marketed for children in Spain are more concentrated $(100 \mathrm{mg} / \mathrm{ml})$ than those marketed in the United States $(32 \mathrm{mg} / \mathrm{ml})$. On the other hand, ALF after therapeutic intake is highly unusual and the mechanism by which it is produced has not yet been determined. In a clinical trial performed in healthy adult volunteers who received 4 gr of paracetamol per day as a single dose or in combination with opioids during 14 days it was registered an increase of GPT values (more than 5 times the upper limit of normal) in more than $19 \%$ of participants compared to $3 \%$ in placebo group ${ }^{(10)}$. In an epidemiological study, the relative risk (RR) of acute liver failure with therapeutic doses of paracetamol was 7 (CI 99\%: 3.3 - 13.9) with an estimated incidence rate of 2.4 cases per 100,000 person-years of exposure ${ }^{(11)}$. In our retrospective study, we found that only 1 of 49 patients with acute poisoning showed hepatotoxicity, whereas 7 of 8 patients with chronic poisoning showed hepatotoxicity, of which 3 met criteria of acute liver failure ${ }^{(8)}$.

Craig et al ${ }^{(12)}$ evaluated hospitalized patients admitted for impairment of liver function secondary to paracetamol intake between 1992 and 2008, obtaining 663 patients, of whom $161(24.3 \%)$ had chronic use of this drug (defined as the intake of 2 or more supratherapeutic doses of paracetamol over a time interval of more than 8 hours, resulting in a cumulative dose greater than 4 grams per day). It was evidenced that patients with chronic intake were more likely to develop hepatic encephalopathy at any time of clinical evolution than patients with acute ingestion. Similar results were obtained in the study conducted at our hospital, where retrospectively evaluated cases with suspected paracetamol intake in both adults and children, it was observed that patients with chronic paracetamol intake were at higher risk of developing ALF than patients with acute ingestion ${ }^{(8,13)}$.

The role of metabolism in the mediation of toxicity has been recognized since the 1970s and has been studied in animal models to understand the mechanism of paracetamol hepatotoxicity ${ }^{(14-16)}$ where oxygen and nitrogen stress are considered important in mediating toxicity ${ }^{(17-21)}$. On the other hand, biochemical and ultrastructural studies have shown that toxic doses of paracetamol could cause changes in the morphology and function of hepatic mitochondria ${ }^{(22)}$. Thus, in rodents, binding of the toxic metabolite to proteins leads to mitochondrial dysfunction and nuclear DNA fragmentation, resulting in necrotic cell death. This mechanism has been proposed to explain the hepatic toxicity of paracetamol in humans. McGill et al have published this mechanism of hepatotoxicity in humans after intake of supratherapeutic doses of paracetamol ${ }^{(23)}$. It is important to determine the detection of the biomarkers of liver damage in early stage. Metabolic technology (with mass spectrometry and nuclear magnetic resonance) may have the ability to identify specific biomarkers of adverse events and toxicity in the early stages ${ }^{(24)}$. After low doses of paracetamol, $90 \%$ of this drug is metabolized to paracetamol-glucuronide and paracetamol-sulfate in the liver by glucuronyltransferase and sulfotransferase and 
subsequently eliminated through the urine. Conjugation sulfate is the dominant metabolic pathway in neonates and young children, but glucuronid metabolism increases with age and less than $10 \%$ is metabolized by cytochrome P450 to form NAPQI, which is immediately neutralized by conjugation with glutathione and excreted in the urine as cysteine and conjugates of mercapturic acid. In a situation where paracetamol overdose, or in circumstances where there is a reduction of glutathione, the metabolite NAPQI is covalently bound to the cellular proteins forming adducts ${ }^{(25)}$. James LP et al. recognized that there was a correlation between adducts and clinical markers of hepatotoxicity commonly used in children and adolescents with paracetamol overdose ${ }^{(26)}$. In addition, the adducts were not found in patients with other known causes of acute hepatic failure and in those patients receiving $\mathrm{N}$-acetyl cysteine treatment were able to detect low levels of adducts.

Metabolomics is the science responsible for the detection, identification and quantification of metabolites present in biological samples. The absence or presence of some of these metabolites, as well as the relative concentration between them, may be an indicator of disease states or predisposing factors. In recent decades it has also evolved in the use of transcriptomic technology to understand the mechanism of liver toxicity. Gene expression can provide information that reflects the physiological status of the body. Bushel et al ${ }^{(27)}$ confirmed the hypothesis that gene expression patterns derived from peripheral blood cells could predict the degree of liver injury following an acute overdose of paracetamol before it can be detected by traditional parameters. Analysis of the genes revealed more alteration in the inflammatory pathways involving IL-1 and NF-kB. Treatment with paracetamol has been shown to produce expression in blood cells 48 hours after initiation of treatment without liver damage. The magnitude of these changes in expression correlated positively with the production of NAPQI metabolite and the increase in serum lactate, as demonstrated in metabolomics studies.

The Rumack-Matthew nomogram is widely used and assesses the need to administer the $\mathrm{N}$-acetylcysteine antidote (NAC) after an acute paracetamol poisoning ${ }^{(28)}$. The nomogram was based on data from timedependent paracetamol concentrations in the population with an acute overdose of paracetamol that came to the emergency services within the first 24 hours of the intake. The assessment of the risk of hepatotoxicity is difficult to evaluate in patients who do not meet the following criteria: children, arrival at the emergency department 24 hours after the intake, chronic overdose of paracetamol, the presence of risk factors (dehydration, alcoholism) (29) concomitant user of other medication (particularly enzyme inducers) ${ }^{(30)}$ and patients with sustained release paracetamol formulations ${ }^{(31)}$. An important difference between the nomogram and the adduct-based assessment is that the former focuses on the determination of the levels of the original drug regardless of metabolism, while the adduct levels are a measure of the remaining NAPQI from detoxification by glutathione, as well as the contributions of both phases of metabolism.

The identification of paracetamol toxicity biomarkers could have important clinical implications for groups of patients who can not apply the Rumack-Matthew nomogram. And could be useful in the evaluation of children with acute liver failure of unknown etiology, in addition to predict the occurrence of liver damage in its early stage. On the other hand, it is necessary to perform an evaluation of acute poisoning by the Rumack-Matthew nomogram in these patients.

\section{OBJECTIVES}

1. Primary:

- To determine the clinical factors and biomarkers (pharmacokinetics, genetics and metabolomics) that predicts the development of hepatotoxicity in the paediatric population after acute and chronic paracetamol intake.

- To develop a predictive model to assess the risk of hepatotoxicity in acute and chronic paracetamol overdoses adapted to paediatric patients for use in clinical practice.

\section{Secondary}

- Characterize acute and chronic overdoses of paracetamol, according to demographic characteristics, intake reason, dose, serum paracetamol levels, transaminase values, bilirubin, creatinine, INR, prothrombin activity, phosphate, $\mathrm{pH}$.

- To determine paracetamol pharmacokinetics in paediatric population in acute overdose.

- To study paracetamol pharmacokinetics in paediatric population in chronic overdose.

- To evaluate the clinical and analytical course of chronic paracetamol overdose.

- Create an action protocol in cases of chronic overdoses by paracetamol in the paediatric population.

- Design of a nomogram in pediatric population that could be useful for predicting the risk of hepatotoxicity in this population.

\section{METHODS}

\section{Design and setting}

This study is designed as an observational prospective study (protocol code: GEIPA-2012-01, AEMPS classification: EPA-OD, Study Reference Number of European Network of Centers for Pharmacoepidemiology and Pharmacovigilance (ENCePP: ENCePP/SDPP/3276) with case control for the evaluation of biomarkers of liver damage efficient to detect liver damage in early stages of acute and chronic paracetamol overdose in paediatric population. This study will be performed in La Paz 
University Hospital, Niño Jesus Hospital and Gregorio Marañón University Hospital in Madrid (Spain).

\section{Study population and inclusion criteria}

The target population will be a cohort of paediatric patients under 18 years with suspected paracetamol overdose ingestion who will be treated in the emergency department of La Paz University Hospital, Niño Jesus Hospital and Gregorio Marañón University Hospital with established criteria for suspected overdose of paracetamol, both acute and chronic

\section{Study timeline}

A Flowchart of the study procedures can be found in table 4.

\section{a. Inclusion phase}

Inclusion criteria are (i) male and female patients under 18 years of age who suspected acute overdose ingested in a single dose, fulfilling the criteria shown in table 1, (ii) Male and female patients who suspected chronic overdose ingested, fulfilling the criteria shown in table 2. Patients with a weight less than $6 \mathrm{~kg}$ were excluded. Two control cohorts, matched by age and clinical characteristics will be recruited, fulfilling the criteria shown in table 3 .

\section{b. Result collection and analysis}

An interview will be made to the patient (parent or guardian) during hospitalization. At the same time, blood and urine samples will be collected for pharmacokinetic, genetic and metabolomic analysis. Results will be collected in electronic databases for subsequent statistical analysis.

\section{c. Results dissemination}

- Preparation of a final report.

- Publication of the results in scientific journals.

- Communications in scientific meetings and conferences.

Table 1. Acute overdose criteria

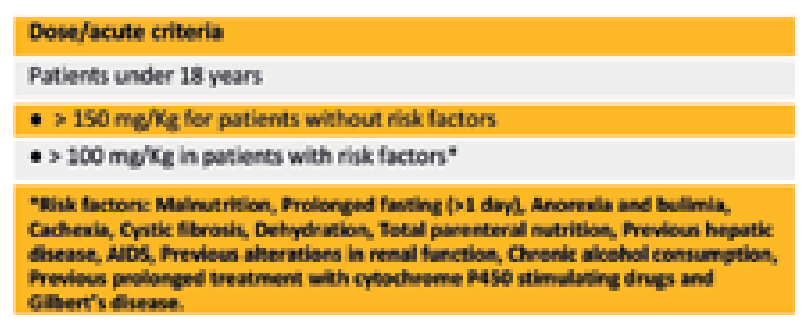

\section{Data collection}

Patient's clinical information will be collected by medical history review and a personal interview with patients and relatives. The first samples (point A) will coincide with the determination of paracetamol concentrations, liver and renal function and coagulation upon arrival to the emergency department. In addition, a portion of the blood will be requested for genetic determination and metabolomic analysis (the additional amount will be $2.5 \mathrm{ml}$ ) plus a urine sample for the metabolomic profile. A second sample (point B) for control analysis (blood count, coagulation, liver function and renal function) and paracetamol concentrations, a portion of the blood will be required for metabolomic (the amount of additional blood will be $2 \mathrm{ml}$ ) and urine for metabolomic profile. As part of the clinical control, the patients will be evaluated at 72 to 96 hours after paracetamol intake for a new clinical and analytical assessment. We will request a portion of the third blood sample of analytical control of approximately the same amount as the second $(2 \mathrm{ml})$ and urine for metabolomic (point C). Summary table of blood samples in patients with the lowest weight established to participate in the study $(6 \mathrm{Kgs})$ is shown in table 5 . All blood samples and biomasses will be sent to the Clinical Pharmacology Service (paracetamol toxicity group in children).

Table 2. Chronic overdose criteria

\begin{tabular}{|c|c|}
\hline \multicolumn{2}{|l|}{ Dowe/chronicity critterla } \\
\hline$>6$ veans & Children 0.6 years \\
\hline 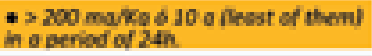 & $\bullet>200 \mathrm{mg} / \mathrm{K}_{\mathrm{g}}$ in o period of $24 \mathrm{~h}$ \\
\hline 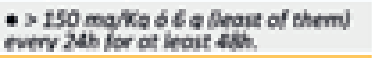 & 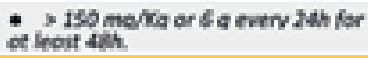 \\
\hline 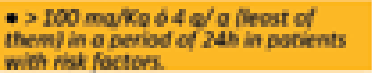 & 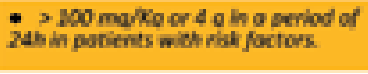 \\
\hline
\end{tabular}

a. Preservation of blood samples for genotyping and genotyping technique.

For pharmacogenetic analysis, a sample of whole blood (in tube with EDTA-K2) will be obtained and stored in a freezer at $-80^{\circ} \mathrm{C}$ located at the Clinical Pharmacology Service. DNA extraction will be performed using the QuickGene DNA blood kit S kit (Fujifilm ${ }^{\circledR}$, Düsseldorf, Germany). Subsequently, allele genotyping will be performed by a customized open array (PharmArray $\left.{ }^{\circledR}\right)$, in the Medical and Molecular Genetic Institute of La Paz University Hospital.

b.Preservation of samples for metabolomic analysis and metabolomic technique.

The metabolomic analysis will be performed from the 3 urine samples and the 3 serum samples and the plasma sample from the point A extraction. The samples will be analyzed and interpreted in the Interdepartmental Research Service at the University of Medicine of the Universidad Autonoma de Madrid.

\section{Case Report Form (CDR)}

Each patient's CDR will include the following information:

- Age, sex, ethnic group and weight of the patient.

- Date and time of admission to paediatric emergencies.

- Time elapsed from paracetamol intake to arrival in the emergency service.

- Presence of risk factors (Malnutrition, prolonged fasting ( $>1$ day) anorexia-bulimia, cachexia, cystic fibrosis, present dehydration, total parenteral nutrition, previous liver disease, AIDS, previous alterations of renal function, chronic alcohol consumption, previous prolonged treatment with drugs that stimulate 
cytochrome P450, Gilbert's disease).

- Dosage of paracetamol ingested, dietary intake (acute or chronic).

- Formulation of paracetamol ingested.

- Other drugs ingested in the last 15 days, start date and end date.

- Analytical Findings: GPT/ALT (UI/L), GOT/

AST, GGT (UI/L), Total Bilirubin (mg/dl), Alkaline phosphatase (IU/L), Prothrombin Activity (\%), INR, Glucose (mg/dl), Lactic acid (mg/dl), Phosphate (mg/dl), Serum creatinine $(\mathrm{mg} / \mathrm{dl})$.

- Treatment received and time elapsed (since ingestion): N-Acetylcysteine, gastric lavage, activated carbon.

- Symptoms prior to paracetamol intake: fever, pain, other.

- Symptoms after paracetamol intake: abdominal pain, nausea, vomiting, decay, altered level of consciousness, irritability.

- Risk assessment of hepatotoxicity by Rumack-Matthew nomogram: yes or no.

- Result of Rumack-Matthew Nomogram.

- If Rumack-Matthew nomogram could not be applied, explain why.

- Outcome of the hospitalization: discharge, transfer (motive), exitus, transplant.

Table 3. Controls criteria

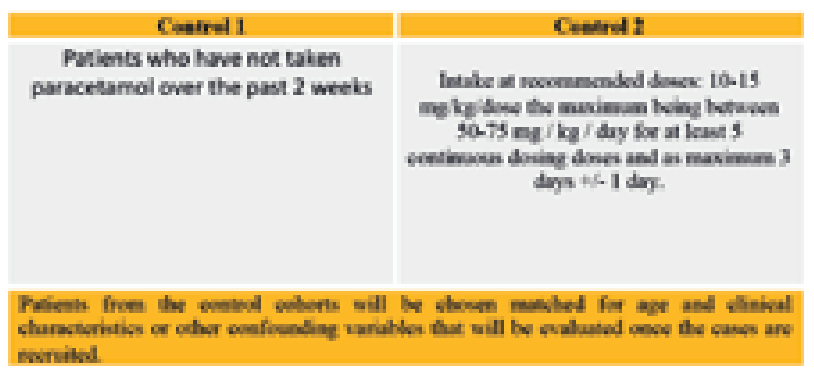

\section{Withdrawal criteria}

Patients may discontinue their participation in the study at any time. The research doctor, in his or her opinion or judgment, may also withdraw a patient from the study if required by the patient's clinical situation or if the patient does not comply with the protocol. The cause and justification of the withdrawal will be reflected in the study development.

\section{Sample size calculation}

Accepting an alpha risk of 0.05 and a beta risk of 0.2 in a bilateral contrast, al least 36 cases and 144 controls (72 controls 1, 72 controls 2 ) are required to detect a minimum odds ratio of 3 . It is assumed that the rate of exposure in the control group will be 0.4. A followup loss rate of $0 \%$ has been estimated. The POISSON approximation has been used.

\section{Data análisis}

- Statistical analysis of the results:

Frequency results will be expressed in absolute terms,
Table 4. Study Development

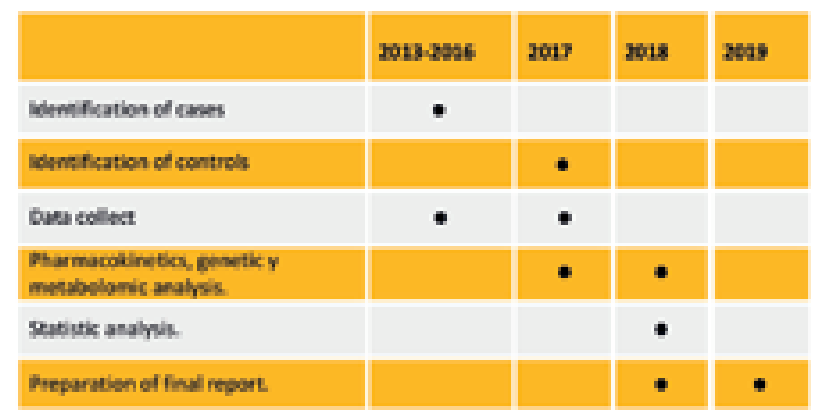

such as percentages and confidence intervals. Continuous variables will be expressed as mean (SD) and median (range) according to normality test (Kolmogorov Smirnov test). For the estimation of differences between variables Chi square or Student's T will be used if they are parametric. In case of not following a normal distribution, non-parametric tests (U of Mann-Whitney or Kruskal Wallis, as appropriate) will be used. The data will be analyzed using the statistical computing environment R (R Development Core Team, 2013) and RStudio. To determine the factors associated with liver damage, a multivariate logistic regression will be performed in which the dependent variable will be the presence of hepatotoxicity (following previously defined parameters) and as independent factors: demographic, clinical, analytical, kinetic, genetic and metabolomic factors. - Likewise, multivariate linear regression will be used to determine the relationship between transaminases values and factors (demographic, clinical, kinetic, analytical, and genetic).

For the development of the nomogram, at least two samples per patient will be collected to determine the level of paracetamol in relation to the hours elapsed since ingestion, a curve is created that relates both parameters and through a ROC curve determine the cutoff point for greater specificity and sensitivity.

\section{- Causality analysis}

In those cases where hepatic function is impaired, a causality analysis will be carried out using the algorithm of the Spanish Pharmacovigilance System ${ }^{(32)}$ and CIOMS (33) to detect cases attributable to paracetamol.

\section{Ethical aspects}

Researchers are strictly attending to the provisions of this protocol and the standards of good clinical practice. The European Community guidelines included in the fourth section of chapter V of "Notice to Applicants" and "Notice to Marketing Authorization Holders" shall be followed. Pharmacovigilance

Guidelines “of 29 January 1999, RD 1344/2007, of 11 October, regulating the pharmacovigilance of medical products for human use, as well as the applicable aspects of the Spanish Medicine Agency's circular $n^{\circ}$. 15/2002 in accordance with Royal Decree 711/2002.

Following the guidelines on observational studies for 
Table 5. Summary table of blood samples in patient with the lowest weight established to participate in the study (6 Kgs).

\begin{tabular}{|c|c|c|c|c|c|}
\hline \multirow[t]{2}{*}{ Exreactions: } & \multicolumn{3}{|c|}{ 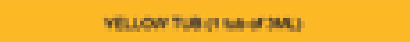 } & \multirow{2}{*}{$\begin{array}{l}\text { ruper } \\
\text { the iti } \\
\text { osen. }\end{array}$} & \multirow{2}{*}{ 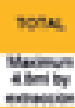 } \\
\hline & moosters & mentus & 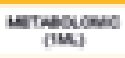 & & \\
\hline a & 1 & 92 & 6 & 98 & is \\
\hline . & 1 & 92 & 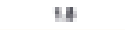 & & , \\
\hline 5 & 1 & 92 & 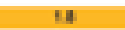 & & 3 \\
\hline 10tais & 3 & 40 & 84 & 03 & is \\
\hline
\end{tabular}

medicinal products for human use, the study will be evaluated by the Ethical Committee of Clinical Research of the La Paz University Hospital. The current revision of the Helsinki declaration that can be consulted in World Medical Association website (http://www.wma. net/en/30publications/10 policies/b3/index.html) is the accepted basis for ethics in human research and must be scrupulously followed and respected by all persons involved in such research.

Only the researchers will know the data (initials of the name of the patient and number of clinical story) that can identify the patients. The initials of the names and the patient's history number are separated from the rest of the case report form, and there are no entries entered into the study database. The patient will be identified by a numerical code in order to respect the confidentiality of patient's personal data, as established in Organic Law 15/1999 on Personal Data Protection. The collection and handling of samples will follow the indications established in Law 14/2007, of July 3, on biomedical research.

\section{DISCUSSION}

The detection of hepatotoxicity during the drug development phase is complicated. One of the reasons is that an efficient biomarker of liver damage is not available to detect liver injury in the early stages ${ }^{(34)}$. Alanine aminotransferase values have been traditionally used to diagnose patients with acute liver injury after paracetamol intoxication; however, in acute overdose, the elevation of this biomarker may be delayed $48-72 \mathrm{~h}$ after ingestion, hindering prompt recognition of the overdose. Thus in recent years, there has been research into the presence of other biomarkers that could effectively and promptly predict the occurrence of liver damage before ALT elevation. Antoine et al., $2013^{(35)}$ found 3 possible biomarkers: elevation of microRNA-122, a marker of necrosis (HMGB-1) and markers of necrosis and apoptosis (K18). On the other hand, studies performed by the covalent junctions that form between NAPQI and cellular proteins can be used to identify patients with occult toxicity by paracetamol ${ }^{(26,36)}$. The metabolic technology (with mass spectrometry and nuclear magnetic resonance) may have the ability to identify specific biomarkers of adverse events and toxicity in the early stages ${ }^{(24)}$. The development of these new biomarkers focuses on the metabolic pathways of paracetamol and the mechanisms responsible for its toxicity. However, despite the research, the molecular and cellular events associated with an overdose of paracetamol are not completely understood and the toxicity of this drug remains an unresolved clinical problem. The development of new biomarkers of paracetamol toxicity could be useful in the diagnosis and/or management of these patients. The objective of the present study is the determination of new biomarkers that are specific to the overdose of paracetamol and may be useful in the evaluation of children with acute liver of unknown etiology, in addition to being able to predict the appearance of hepatic injury in its early stage.

On the other hand, it is necessary to make an evaluation of the acute overdose of this drug in the paediatric population and to verify the usefulness of the RumackMatthew nomogram in these patients. It is known that the nomogram was based on data from time-dependent paracetamol concentrations in adults with a single intake of paracetamol that came to the emergency department within the first 24 hours post-ingestion. But this nomogram has been not assessed in children, lateonset (> 24 hours after ingestion), chronic paracetamol intake, concomitant intake of others drugs that may alter the pharmacokinetics of paracetamol, and patients with intakes of prolonged release paracetamol formulations. We think that it is necessary to perform an evaluation of Rumack-Matthew nomogram in these patients.

Our data indicate that from a pragmatic standpoint, the chronic toxicity cases in children should be taken into greater consideration. The administration of the antidote is effective in the first 8 hours following poisoning; thereby the risk of hepatotoxicity is significant in patients with chronic poisoning because N-Acetylcysteine is less effective for late-presenting patients. Mahadevan et al ${ }^{(37)}$ found that late arrival to the hospital following a paracetamol poisoning was a risk factor for developing severe hepatotoxicity, thereby increasing mortality. The relationship between chronic ingestion and hepatic toxicity revealed that these episodes are less frequent than acute poisoning, but the potential risk is higher (a significant OR of 65.93 for hepatitis and 28.50 for acute liver failure). The increased severity observed in chronic poisoning might be due, among other factors, to the poor perception by parents/guardians of the high risk of hepatotoxicity associated with the repeated consumption of supratherapeutic doses. This lack of perception is evidenced by the delay in seeking medical help, a delay that is greater in chronic consumers than non-chronic consumers. Also, if physicians did not suspect paracetamol poisoning enough or if they use the Rumack- Matthew nomogram to decide the risk, it will be too late to avoid severe hepatotoxicity.

The present study began in 2012, by the time of publication of this article; 37 cases have already been recruited, of which 32 for acute intoxication and 5 for chronic intoxication.

We expect that the results of this study can help to improve 
the diagnosis and the evolution of these patients.

\section{DISCLOSURES}

This research has not received specific grant from any funding agency in the public, commercial, or not-forprofit sectors. The authors declare that they have no conflicts of interest.

\section{REFERENCES}

[1]. Larson AM, Polson J, Fontana R J, Davern TJ, Lalani E, Hynan LS, et al. Acute Liver Failure Study Group. Acetaminophen-induced acute liver failure: results of a United States multicenter, prospective study. Hepatology 2005;42:1364-1372.

[2]. Bernal W. Changing patterns of causation and the use of transplantation in the United Kingdom. Semin Liver Dis 2003;23:227-237.

[3]. Wei G, Bergquist A, Broomé U, Lindgren S, Wallerstedt S, Almer S, et al. Acute liver failure in Sweden: etiology and outcome. J Intern Med 2007;262:393-401.

[4]. Moore T, Cohen M, Furberg C. Serious adverse drug events reported to the Food and Drug Administration, 1998-2005. Arch Intern Med 2007;167:1752-1759.

[5]. Squires R, Shneider B, Bucuvalas J, Alonso E, Sokol R, Narkewicz M, et al. Acute liver failure in children: the first 348 patients in the pediatric acute liver failure study group. J Pediatr 2006;148:652-8.

[6]. Narkewicz M, Dell D, Karpen S, Murray K, Schwarz K, Yazigi N, et al. Pattern of Diagnostic evaluation for the causes of pediatric acute liver failure: an opportunity for quality improvement. J Pediatr 2009;155:801-806.

[7]. Mintegi S, Fernández A, Alustiza J, Canduela V, Mongil I, Caubet I, et al. Emergency visits for Childhood Poisoning: A 2 - year prospective multicenter survey in Spain. Pediatr Emerg Care2006;22:334-38.

[8]. Tong HY, Medrano N, Borobia AM, Ruiz JA, Martínez AM, Martín J, et al. Hepatotoxicity induced by acute and chronic paracetamol overdose in children: Where do we stand? World J Pediatr. 2017;13:76-83. doi: 10.1007/s12519-016-0046-6.

[9]. Escorsell A, Mas A, de la Mata M, Spanish Group for the Study of Acute Liver Failure: Acute liver failure in Spain: analysis of 267 cases. Liver Transpl 2007;13:1389-95.

[10]. Watkins PB, Kaplowitz N, Slattery JT, Colonese CR, Colucci SV, Stewart PW, et al. Aminotransferase elevations in healthy adults receiving 4 grams of acetaminophen daily. JAMA 2006;296:87-93.

[11]. Sabaté M, Ibáñez L, Pérez E, Vidal X, Buti M, Xiol $X$, et al. Risk of acute liver injury associated with the use of drugs: a multicentre population Surrey. Aliment Pharmacol Ther 2007;25:1401-09.

[12]. Craig D, Bates C, Davidson J, Martin K, Hayes P, Simpson K. Staggered overdose pattern and delay to hospital presentation are associated with adverse outcomes following paracetamol-induced hepatotoxicity. Br J Clin Pharmacol 2011;73:285-94.

[13]. Tong HY, Medrano N, Borobia AM, Martínez AM, Martín J, Ruiz JA, et al. Hepatotoxicity induced by acute and chronic paracetamol overdose in adults. Where do we stand? Regul Toxicol Pharmacol. 2015;72:370-8.

[14]. Jollow DJ, Mitchell JR, Potter WZ, Davis DC, Gillette JR, Brodie BB. Acetaminophen-induced hepatic necrosis. II Role of covalent binding in vivo. J Pharmacol Exp Ther 1973;187:195-202.

[15]. Mitchell JR, Jollow DJ, Potter WZ, Davis DC, Gillette JR, Brodie BB. Acetaminophen-induced hepatic necrosis I. Role of drug metabolism. J Pharmacol Exp Ther 1973;187:185-94.

[16]. Roberts DW, Pumford NR, Potter DW, Benson RW, Hinson JA. A sensitive immunochemical assay for acetaminophen-protein adducts. J Pharmacol Exp Ther 1987;241:527-33.

[17]. Brand MD, Affourtit C, Esteves TC, Green K, Lambert AJ, Miwa S, et al. Mitochondrial superoxide: production, biological effects, and activation of uncoupling proteins. Free Radic Biol Med 2004;37:755-67.

[18]. Casteilla L, Rigoulet M, Penicaud L. Mitochondrial ROS metabolism: modulation by uncoupling proteins. IUBMB life 2001;52:181-88.

[19]. Koop DR. Oxidative and reductive metabolism by cytochrome P450 2E1. FASEB J. 1992;6:724-730.

[20]. Sies H, de Groot H. Role of reactive oxygen species in cell toxicity. Toxicol Lett. 1992;64-65:547-551.

[21]. Hinson JA, Pike SL, Pumford NR, Mayeux PR. Bitrotyrosine-protein adducts in hepatic centrilobular areas following toxic doses of acetaminophen in mice. Chem Res Toxicol 1998; 11:604-7

[22]. Placke ME, Ginsberg GL, Wyand DS, Cohen SD. Ultrastructural changes during acute acetaminopheninduced hepatotoxicity in the mouse: a time and dose study. Toxicol Pathol. 1987;15:431-438.

[23]. McGill MR, Sharpe MR, Williams CD, Taha M, Curry SC, Jaeschke H. The mechanism underlying acetaminophen-induced hepatotoxicity in humans and mice involves mitochondrial damage and nuclear DNA fragmentation. J Clin Invest2012;122:1574-1583.

[24]. Van Ravenzwaay B, Cunha GC, Leibold E, Looser R, MellerW, Prokoudine A, et al. The use of metabolomics for the discovery of new biomarkers of effect. Toxicol let 2007;172:21-8.

[25]. Streeter AJ, Harvoson PJ, Nelson SD, Baillier TA. Cross-linking of protein molecules by the reactive metabolite of acetaminophen, N-acetil-p-benzoquinona imina, and related quinoid compounds. Adv Exp Med Biol 1986;197:727-737.

[26]. James LP, Alonso EM, Hynan LS, Hinson JA, Davern TJ, Lee WM, et al. Detection of acetaminophen protein adducts in children with acute liver failure of inderteminate cause. Pediatrics 2006;118:e676-e681. 
[27]. Bushel PR, Heinloth AN, Li J, Huang, L., Chou, J. W., Boorman, G. A, et al. Blood gene expression signatures predict exposure levels. Proc Natl Acad Sci. USA 2007;104:18211-18216.

[28]. Rumack B, Mathhew H. Acetaminophen poisoning and toxicity. Pediatrics 1975; 55:871-76.

[29]. Ali FM, Boyer EW, Bird SB. Estimated risk of hepatotoxicity after an acute acetaminophen overdose in alcoholics. Alcohol 2008;42:213-218.

[30]. Halcomb SE, Sivilotti ML, Goklaney A, Mullins ME. Pharmacokinetic effects of diphenhydramine or oxycodone in simulated acetaminophen overdose. Acad. Emerg. Med 2005;12:169-172.

[31]. Bizovi KE, Aks SE, Paloucek F, Gross R, Keys N, Rivas J. Late increase in acetaminophen concentration after overdose of Tylenol Extended Relief. Ann Emerg Med 1996;28:549-551.

[32]. Capellà, D., \& Laporte, J. (1993). La notificación espontánea de reacciones adversas a medicamentos. In J. Laporte \& G. Tognoni (Eds.), Principios de epidemiología del medicamento (pp. 147-170).
Barcelona: Masson-Salvat.

[33]. Bénichou C. Criteria of drug-induced liver disorders. Report of an international consensus meeting. J. Hepatol. 1990 Sep;11(2):272-276.

[34]. Beger R, Sun J, Schnackenberg L. Metabolomics approaches for discovering biomarkers of druginduced hepatotoxicity and nephrotoxicity. Toxicol Appl Pharmacol 2010;243:154-166.

[35]. Antoine DJ, Dear, JW, Lewis PS, Platt V, Coyle J, Masson M, et al. Mechanistic biomarkers provide early and sensitive detection of acetaminophen-induced acute liver injury at first presentation to hospital. Hepatology 2013; 58:777-787.

[36]. Davern TJ 2nd, James LP, Hinson JA, Polson J, Larson AM, Fontana RJ, et al. Measurement of serum acetaminophen-protein adducts in patients with acute liver failure. Gastroenterology 2006;130:687-694.

[37]. Mahadevan SBK, McKiernan PJ, Davies P, Kelly DA. Paracetamol induced hepatotoxicity. Arch Dis Child 2006;91:598-603. 